\title{
Simulation of Flat Surface Modle Film Cooling Enhancement with Different Injection Orientation
}

\author{
http://dx.doi.org/10.3991/ijoe.v9i3.2556 \\ Z.L. $\mathrm{Yu}^{1}, \mathrm{~T}$. $\mathrm{Xu}^{1}$, J.L. $\mathrm{Li}^{1}$ and Y.W. $\mathrm{Li}^{2}$ \\ ${ }^{1} \mathrm{~J}$ ilin University, Changchun, PR China \\ ${ }^{2}$ R\&D Center, FAW, Changchun, PR China
}

\begin{abstract}
The cooling effectiveness of combustor transition pieces increases significantly in accordance with turbine temperature. In this issue, we create a scenario in which using three-dimensional computational to predict the performance of film cooling model with three rows holes and double chambers on the flat surface that the model could simulate the TP's structure and performance. Fluent, a commercial CFD software, is extensively used in the current work for numerical simulations. A comprehensive study is performed on the effect of coolant injection angles on film cooling. The the temperature distribution in the inner wall, cooling effectiveness, and the velocity distribution of coolant flow with different cases is compared. Analysis on flow injection orientations is beneficial to enhance the turbine inlet temperature and improve gas turbine efficiency.
\end{abstract}

Index Terms - film cooling, flat surface, cooling effectiveness, coolant flow orientation

\section{INTRODUCTION}

A major challenge associated with gas turbine design is developing methods to cool combustor components so that higher gas temperatures and thus better performance can be achieved. Film cooling becomes increasingly important in aerospace industry in the cooling of hot components. Here we must consider the efficiency of cooling technology which plays significant role in the whole process that the more efficiency it applied; the higher temperature of combustor could exit and the efficiency of gas turbine cycle could reach. Many experimental and computational studied have been conducted in order to study the cooling process of gas turbine blades [1], understand this complex flow and heat process, and devise the best possible cooling schemes. This operating temperature is far above the permissible temperature of currently available thermal-resistive materials. Therefore, a cooling technique has to be established to prevent the thermal degradation of turbine components.

There are numerous parameters affecting the cooling performance, such as the film hole internal fluid dynamics, the turbulence and vortices production, the approaching flows prior to hole entry, the hole shaping, the hole inclination and orientation, the hole spacing and length-todiameter ratio, the density ratio, the blowing ratio, the momentum flux ratio and the mainstream turbulence intensity, the mainstream acceleration, the external surface curvature and external surface roughness. The thermalflow structures, the effectiveness of the adiabatic film cooling, and the heat transfer rate for various discrete-hole geometries with flat-plate or constant-curvature models have been documented [2-6]. This trend of active research is continuing, striving for new techniques to improving film cooling performance so that it can be an ideal tangential slot injection [7]. Majority of the literature has been covered in the book by Han et al. [8]. There have been several studies about shower head film cooling in the past; however most of these studies concentrate on blade shower head film cooling, my essay shall be starting from the transform piece and the model illustrate is double chambers film cooling.

While a large amount of coolant provides better surface coverage downstream of the jets, using too much coolant, on the other hand, can incur a severe efficiency penalty. In this issue, the double chamber model with the flat surface is simulating the structure of transition piece, which has rarely mentioned in most studies. The main objective of this thesis is to elucidate the film-cooling effectiveness by changing coolant injection angular with three staggered rows of simple round holes in the double chamber model, as determined by using the CFD technique. Accordingly, the main characteristics of this investigation are as follows: (1) Realistic, discrete-hole film-cooling flat surface with three rows of staggered injection coolant intercalation are studied. (2) The coolant injection angle on film cooling effectiveness over the flat surface are investigated. (3) The temperature of inner wall, distributions of coolant velocity, and film-cooling effectiveness contours in different conditions are compared.

\section{NUMERICAL METHOD}

The present film cooling study involves flow which is steadied, Newtonian, three-dimensional, incompressible and turbulent. Such flow behaves according to three fundamental laws, namely the laws of continuity, conservation of momentum, and conservation of energy [9].

The realizable $k-\varepsilon$ model proposed by Shih et al. [10] was intended to address these deficiencies of standard $k-\varepsilon$ models by adopting the following: (1) contains a new formulation for the turbulent viscosity; and (2) a new transport equation for the dissipation rate, $\varepsilon$, is derived from an exact equation for the transport of the mean square vorticity fluctuation. The modeled transport equations for $\mathrm{k}$ and $\varepsilon$ in the realizable $k-\varepsilon$ model are:

$$
\begin{aligned}
\frac{\partial}{\partial x_{i}}\left(\rho k u_{i}\right)= & \frac{\partial}{\partial x_{j}}\left[\left(\mu+\frac{\mu_{t}}{\sigma_{k}}\right) \frac{\partial k}{\partial x_{j}}\right]+G_{k}+G_{b} \\
& -\rho \varepsilon-Y_{M}+S_{k}
\end{aligned}
$$




$$
\begin{aligned}
\frac{\partial}{\partial x_{i}}\left(\rho \varepsilon u_{i}\right)= & \frac{\partial}{\partial x_{j}}\left[\left(\mu+\frac{\mu_{t}}{\sigma_{\varepsilon}}\right) \frac{\partial \varepsilon}{\partial x_{j}}\right]+\rho C_{1} S_{\varepsilon}- \\
& \rho C_{2} \frac{\varepsilon^{2}}{k+\sqrt{v \varepsilon}}-C_{1 \varepsilon} \frac{\varepsilon}{k} C_{3 \varepsilon} G_{b}+S_{\varepsilon}
\end{aligned}
$$

where

$$
\begin{aligned}
C_{1} & =\max \left[0.43, \frac{\eta}{\eta+5}\right], \\
\eta & =S \frac{\varepsilon}{k} \\
S & =\sqrt{2 S_{i j} S_{i j}}
\end{aligned}
$$

In these equations, $G_{k}$ and $G_{b}$ represent the generation of turbulent kinetic energy due to the mean velocity gradients and buoyancy, respectively. $Y_{M}$ is the contribution of the fluctuating dilatation in compressible turbulence to the overall dissipation rate, $C_{1 \varepsilon}$ and $C_{2}$ are constants. $S_{k}$ and $S_{\varepsilon}$ are user-defined source terms. The turbulent (or eddy) viscosity $\mu_{t}$ is computed by combining $\mathrm{k}$ and $\varepsilon$ as follows:

$$
\mu_{t}=\rho C_{\mu} k^{2} / \varepsilon
$$

A benefit of the realizable $k-\varepsilon$ model is that it better predicts the spreading rate of both planar and round jets. It is also stated that it has superior performance for flows involving rotation, separation, recirculation and boundary layers under adverse pressure gradients.

\section{A. Thermodynamic equation}

The adiabatic cooling effectiveness $(\eta)$ is used to examine the performance of film cooling. The definition of $\eta$ is

$$
\eta=\frac{T_{m}-T_{a w}}{T_{m}-T_{c}}
$$

where $T_{m}$ is the mainstream hot gas inlet temperature, which is a fixed value for calculation the adiabatic cooling effectiveness of any location, $T_{c}$ is the temperature of the coolant, which is assigned as a constant of $300 \mathrm{~K}$ in this issue. $T_{a w}$ is the adiabatic wall temperature.

\section{PRoBlem Definition}

\section{A. CFD model}

The new transition piece features a rounded body shape that balances the heat transfer loading both internally and externally and eliminates resonant frequency concerns. It has an upstream aperture for the gas flow (which is cylindrical) and it is used to receive the gas flow directly from the corresponding combustion liners with a high level of enthalpy, they are conjured in a longitudinal direction so that their downstream ends comprise arched segments to form a ring-type configuration which opens toward the first stage of the gas turbine (stator) [11]. This study builds a model, double chamber flat plate, which could simulate the TP's structure and performance, to discuss the effect of the type of the coolant injection angle on the film-cooling effectiveness over a flat surface.
A schematic of the flow domain along with boundary conditions and dimensions is given in figure 1. As shown in figure, the model has two chambers with length of 1000 $\mathrm{mm}$ and width of $180 \mathrm{~mm}$. The uperside chamber in the pic is called coolant chamber as the side is closed. Contrarily, the inner chamber goes by the name of mainstream chamber as the gas could through it from one side to another. And the mainstream and coolant chamber height is $37 \mathrm{~mm}$ and $84 \mathrm{~mm}$, respectively. There is a total of 9 holes uniformed distribution in three rows on the surface of the outer wall, and the distance between the two rows of the holes is $50 \mathrm{~mm}$. The size of all the holes is about $10.26 \mathrm{~mm}$.

\section{B. Boundary conditions}

Boundary conditions were applied to specific faces within the domain to specify the flow and thermal variables that dictate conditions within the model. They are a critical constituent to the simulation, and it is important that they are specified appropriately. Boundary conditions are first applied in Gambit, before exporting the mesh film to Fluent. Figure 2 shows the boundary conditions used for the modeling. Respectively, the cooling air and gas is coursing along the cooling chamber and the mainstream chamber with the the opposite direction. In the cooling chamber, the simulation is performed using air as the cooling fluid, velocity and temperature contours are set on the jet holes, pressure on the exit mouth. In another chamber, assume that the mainstream is a mixture of $\mathrm{O}_{2}, \mathrm{H}_{2} \mathrm{O}$, $\mathrm{CO}_{2}, \mathrm{~N}_{2}$, as well as some rare gas, and their ingredients are installed with $9 \%, 8 \%, 13 \%, 69 \%, 1 \%$, respectively. Gas velocity and temperature contours are set on the surface of the sector section, pressure on the exit mouth and natural convection on the outside wall of the model are considered as boundary condition [12].

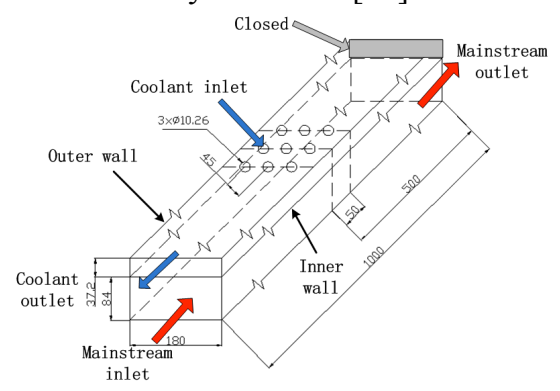

Figure 1. Computational domain showing boundary conditions

TABLE I.

BOUNDARY CONDITIONS

\begin{tabular}{|c|c|c|}
\hline Component & Boundary conditions & Magnitude \\
\hline \multirow{4}{*}{ Mainstream inlet } & Mass flux rate & $31.46(\mathrm{~kg} / \mathrm{s})$ \\
\cline { 2 - 3 } & Gas temperature & $1600(\mathrm{~K})$ \\
\cline { 2 - 3 } & Turbulent intensity & $5(\%)$ \\
\cline { 2 - 3 } & Hydraulic diameter & $0.324(\mathrm{~m})$ \\
\hline \multirow{4}{*}{ Mainstream outlet } & Pressure & $1.512(\mathrm{MPa})$ \\
\cline { 2 - 3 } & Turbulent intensity & $5(\%)$ \\
\cline { 2 - 3 } & Hydraulic diameter & $0.324(\mathrm{~m})$ \\
\cline { 2 - 3 } & Convection coefficient & $10\left(\mathrm{~W} / \mathrm{m}^{2} \mathrm{~K}\right)$ \\
\hline \multirow{4}{*}{ Coolant chamber } & Air temperature & $300[\mathrm{~K})$ \\
\cline { 2 - 3 } & Pressure recovery coefficient & $1.4552(\mathrm{MPa})$ \\
\cline { 2 - 3 } & Turbulent intensity & 0.95 \\
\cline { 2 - 3 } & Hydraulic diameter & $0.01026(\mathrm{~m})$ \\
\cline { 2 - 3 } & & \\
\cline { 2 - 3 } & Pressure & $0.95)$ \\
\hline
\end{tabular}


In this paper, seven injection orientations, $90^{\circ}, 75^{\circ}, 60^{\circ}$, $45^{\circ}, 30^{\circ}, 15^{\circ}$ and $0^{\circ}$, were used in the model. As stated earlier, the mainstream temperature is set at $1300 \mathrm{~K}$ for mainstream and $300 \mathrm{~K}$ for coolant flow. Tables 1 shows a summary of the boundary conditions in the quarter torus and thermal properties of the fluid, respectively.

\section{Meshing and Simulation Procedures}

The computational domain incorporates the model, the tetrahedral mesh in the software, ICEM/CFD, used to generate the structured multi-block and the body-fitted grid system. In this study, the grid system associated with the parts of the mainstream and the coolant supply plenum is a tetrahedral structure. Figure 2 shows the grids of the computational domain. The total number of the cells for the $3 \mathrm{D}$ domain is 467,000 .

This study uses a commercial CFD code based on the control-volume method, ANSYS 12.0.16. All runs were made on a PC cluster with four Pentium-4 $2.8 \mathrm{GHz}$ personal computers. The convergence criteria of the steadystate solution are judged by the reduction in the mass residual by a factor of 6 , typically, in 2000 iterations.

\section{RESULTS AND DISCUSSION}

\section{A. Inner wall temperature}

Figure 3 shows a comparison of temperature distribution on the inner wall with different coolant flow injection orientations, which is the conventional model in actual situations. This region will be the main region of concern in this study. In Figure 3 and Figure 4, temperature and film cooling effectiveness are plotted against the distance along the inner wall between $50 \mathrm{~mm}$ and $750 \mathrm{~mm}$.

The figure illustrates that the temperature at the starting point of the wall is high. It gradually decreases in the downstream. The starting point of the wall is cooled by the coolant holes at the $X=450 \mathrm{~mm}$ on the outer wall. Temperature is the same throughout the coolant holes. After the coolant strikes the inner wall, there are vortexes formed. The jet impingement and the vortex formed out of the coolant flow cools the surface of the inner wall. The delicate color region (orange, yellow and green region) is approximately the region where the coolant strikes the inner wall after being reflected by the coolant holes, which the inner wall is cooled purely by film cooling. The coldest part is at the center of the domain $(X=500 \mathrm{~mm})$. Though almost all flow in the coolant chamber is coolant flow, the cooling depletes along the $\mathrm{X}$ axis, which is why there is a temperature rise in the region far from the impingement spot.

From the figure it is seen that three rows of jet can protect the inner wall from over-heating. With the cooling injection angle increase, the color of temperature distribution in the same region is lighter, and the surface has been better protected by the coolant flow. One important factor to be considered in the design of the inner wall is the inclination of coolant holes. They should be placed in such a way that the cooling flow coming out of the holes should from a uniform film that can cover a large area in the downstream.

\section{B. Cooling effectiveness}

Film cooling performance is measured by the dimension parameter "adiabatic wall effectiveness $(\eta)$ " (equa- tion (5)). Figure 4 presents the centerline and the laterally averaged film cooling effectiveness performance of the hybrid scheme at different coolant injection angular, respectively. It is seen that the effectiveness is high at the start of the film and reduces gradually in the downstream. It is seen from the figure that the effectiveness is maximum between the $X=480 \mathrm{~mm}$ and $620 \mathrm{~mm}$ of the inner wall, which part is under the film region of the outer wall. In the coolant chamber, the flow is predominantly coolant flow and recirculation flow from the vortex that is formed out of the coolant flow. These flows have a low temperature, and thus the effectiveness is high in this region. In the region far from the impingement spot, there is no new coolant injection for heat transfer between coolant and hot wall in the domain, which results in rise of the temperature of the inner wall. Thus, the effectiveness is reduced to below 0.02 in this region. Film cooling effectiveness increases with injection angle, which is because more cooling air is directly reached the inner wall surface and provides better cooling. The figure also shows that the color of coolant effectiveness distribution in the same region is deeper with the cooling injection angle increase, and the surface has been better protected by the coolant flow.

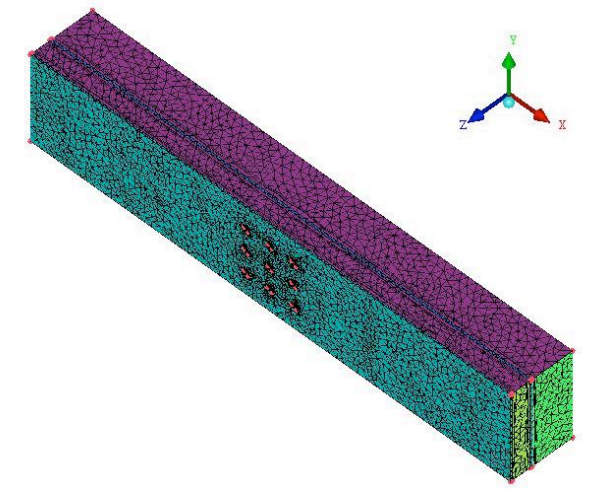

Figure 2. Meshes

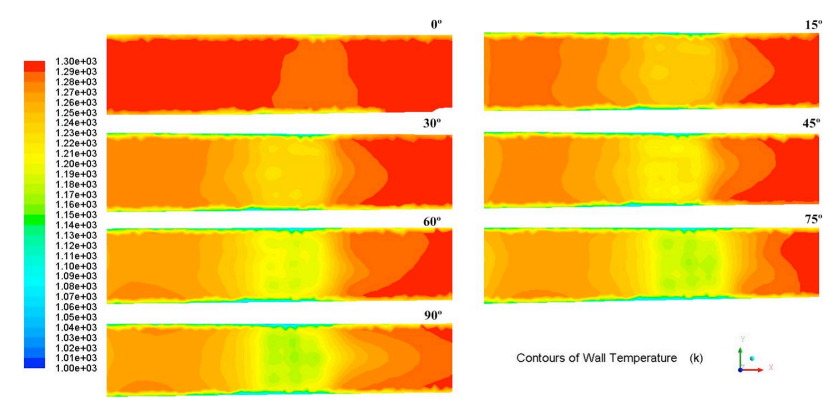

Figure 3. Temperature contours at different coolant injection angles showing vortex induction towards the inner wall

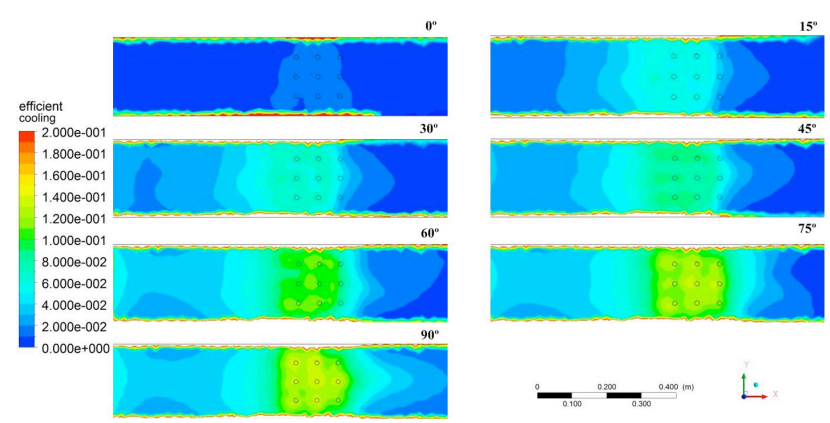

Figure 4. Comparative analysis of cooling effectiveness in different injection angle cases 


\section{Characteristics of the coolant flow}

In order to better understand the mechanisms that lead to improved cooling effectiveness, a sequence of instantaneous images of the coolant flow velocity along the $\mathrm{X}$ axis at different locations with different injection angles are presented in figure 5. The figure illustrates that, as the angle increases, the coolant jet impinges the inner wall surface. This effect shifts the vortex that is formed between two rows into the downstream. This does not allow film cooling to form uniformly throughout the surface of the inner wall, and the coolant flow closer to the inner wall. Therefore, with the injection angles increases, the $90^{\circ}$ angle case has a best cooling performance in the downstream region compared to other cases.

\section{CONCLUSION}

This study explored film cooling effectiveness of a double chamber model with flat surface. Numerical simulation is employed with seven injection angles, $90^{\circ}, 75^{\circ}$, $60^{\circ}, 45^{\circ}, 30^{\circ}, 15^{\circ}$ and $0^{\circ}$, were used in the flat model. The issue is used Fulent to research the cooling effects of film cooling and the temperature distribution in the inner wall, and the velocity distribution of coolant flow. During this investigation, enhanced injection orientation are the reasons for the greater coolant of the transition piece structure.

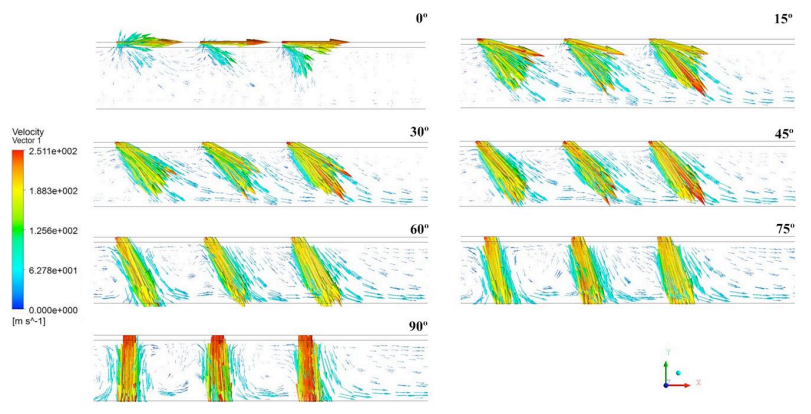

Figure 5. Comparative analysis of coolant flow velocity in different injection angle cases

\section{NOMENCLATURE}

\begin{tabular}{|ll|}
\hline \multicolumn{2}{|l|}{ Nomenclature } \\
\hline$D_{a}$ & diameter of coolant chamber \\
\hline$D_{g}$ & diameter of coolant chamber \\
\hline$L$ & length of the model \\
\hline$T$ & absolute static temperature \\
\hline$X, Y, Z$ & $\begin{array}{l}\text { non-dimensional coordinates in diameter, spanwise } \\
\text { and mainstream directions }\end{array}$ \\
\hline Greek symbols & \\
\hline$\alpha$ & hole angle \\
\hline$\beta$ & Injection angle \\
\hline$\eta$ & film cooling effectiveness \\
\hline Suffixes & mainstraim flow \\
\hline $\mathrm{m}$ & coolant flow \\
\hline $\mathrm{c}$ & adiabatic wall \\
\hline aw & \\
\hline
\end{tabular}

\section{REFERENCES}

[1] Y.P. Lu, D. Allison, and S.V. Ekkad, "Turbine blade shower head film cooling:Inuence of hole angle and shaping," International Journal of Heat and Fluid Flow, vol. 28, pp. 922-931, 2007. http://dx.doi.org/10.1016/j.ijheatfluidflow.2007.01.002

[2] C. Saumweber, and A. Schulz, "Interaction of film cooling rows: effects of hole geometry and row spacing on the cooling performance downstream of the second row of holes," ASME J. Turbomachinery, vol. 126, pp. 237-246, 2004. http://dx.doi.org/10.1115/1.1731395

[3] R.J. Godstein, R.G. Eckert, and J.W. Ramsey, "Film cooling with ingection through holes: adiabatic wall temperatures downstream of a circular hole," Journal of Engineering for Power, Series A, vol. 90, pp. 384-395, 1968.

[4] B. Sen, D.L. Schmidt, and D.G. Bogard, "Film cooling with compound angle holes: heat transfer," ASME J. Turbomachinery, vol. 118, pp. 800-806, 1996. http://dx.doi.org/10.1115/1.2840937

[5] A.K. Sinha, D.G. Bogard, and M.E. Crawford, "Film-cooling effectiveness downstream of a single row of holes with variable density ratio," ASME J. Turbomachinery, vol. 113, pp. 442-449, 1991. http://dx.doi.org/10.1115/1.2927894

[6] R.J. Godstein, and P. Jin, "Film cooling downstream of a row of discrete holes with compound angle". ASME J. Turbomachinery, vol. 123, pp. 222-230, 2001. http://dx.doi.org/10.1115/1.1344905

[7] R.S. Bunker, "Technology Review: A review of shaped hole turbine film-cooling technology," Journal of Hat Transfer, vol. 121, pp. 441-453, 2005. http://dx.doi.org/10.1115/1.1860562

[8] J.C. Han, S. Dutta, and S.V. Ekkad, "Gas Turbine Heat Transfer and Cooling Technology," Taylor and Francis, New York, 2000.

[9] Fluent Inc., Fluent 6.3.26 Documentation, 2003.

[10] T.-H. Shih, W.W. Liou, A. Shabbir, Z. Yang, and J. Zhu, "A new $k-\varepsilon$ eddy viscosity model for high Reynolds number turbulent flows - model development and validation," Computers and Fluids, vol. 24(3), pp. 227-238, 1995. http://dx.doi.org/10.1016/00457930(94)00032-T

[11] J. Benoit, C. Johnston, and M. Zingg, "Enhancing gas turbine power plant profitability: Chronic transition piece and turbine part failures in some $501 \mathrm{~F}$ gas turbines led to a replacement part redesign," Power Engineering, vol. 111(11), pp. 140-144, 2007.

[12] J.A. Alfaro-Ayala, A. Gallegos-Muñoz, J.M. Riesco-Avila, A. Campos-Amezcua, M.P. Flores-López and A. Mani-Gonález, "Analysis of the flow in the combustor transition piece considering the variation in the fuel composition," Journal of Thermal Science and Engineering Applications, vol. 3(2), pp. 245-257, 2011.

\section{AUTHORS}

Z. L. Yu, college of Mechanical Science \& Engineering, Jilin University, Changchun 130025, PR China (email: yuz111@mails.jlu.edu.cn).

T. Xu, college of Mechanical Science \& Engineering, Jilin University, Changchun 130025, PR China.

J. L. Li, college of Mechanical Science \& Engineering, Jilin University, Changchun 130025, PR China (e-mail: j1li11@mails.jlu.edu.cn).

Y. W. Li, R\&D Center, FAW, Changchun 130011, PR China.

This research is supported by the Technology Development of Jilin Province (No. 20126001) and Technology Innovation Project of FAW (No.1332A). Manuscript received 03 March 2013. Published as resubmitted by the authors 12 June 2013 . 